\title{
Predicción de nieve en los modelos Harmonie-Arome y ECMWF
}

\author{
Samuel Arístides Viana Jiménez ${ }^{1}$ (svianaj@aemet.es) \\ Javier Calvo Sánchez1 (fcalvos@aemet.es)
}

${ }^{1}$ AEMET / Departamento de Desarrollo y Aplicaciones / Área de Modelización

\begin{abstract}
RESUMEN
El análisis y la predicción de la nieve han mejorado mucho recientemente debido a la mejora de los análisis, la utilización de parametrizaciones físicas más sofisticadas que incluyen variables de predicción para las partículas de agua condensada incluyendo la nieve y al aumento de la resolución de los modelos. De todas formas, la predicción de la nieve sigue siendo complicada ya que además de la incertidumbre asociada a la predicción de la precipitación, está la incertidumbre asociada con la predicción de la temperatura que en nuestro entorno suele estar próxima al umbral de temperatura que da lugar a precipitaciones en forma de nieve.

En este trabajo se describen las principales características de los esquemas de nieve y los sistemas de asimilación para la nieve en superficie en los modelos del Centro Europeo (ECMWF) y Harmonie-Arome. Además se evalúan las predicciones de ambos modelos, comparando con observaciones in situ y productos de satélite correspondientes a la temporada invernal 2017-2018.
\end{abstract}

PALABRAS CLAVE: nieve; modelo numérico de predicción; cobertura de nieve; espesor de nieve; validación; verificación.

\section{ESQUEMAS DE NIEVE EN SUPERFICIE EN LOS MODELOS HARMONIE-AROME Y ECMWF}

En los modelos numéricos de predicción, la precipitación en forma de nieve se convierte en una variable de superficie una vez que alcanza el suelo, y como tal su evolución pasa a estar controlada por el modelo de superficie correspondiente, que en el caso de Harmonie-Arome es el modelo SURFEX (SURface EXternaliseé; MASson et al., 2013), y para el ECMWF es el TESSEL (Tiled ECMWF Scheme for Surface Exchanges over Land; BALSAMO et al., 2009). En ambos casos, la nieve en superficie se representa mediante esquemas de una sola capa. Su espesor en cada punto viene determinado por el equivalente acuoso de nieve $W_{s}$ y la densidad $\rho$. El equivalente acuoso de nieve evoluciona en función de los aportes recibidos (la precipitación en forma de nieve $\left.P_{s}\right)$ y las pérdidas por fusión $\left(M_{l t}\right)$ y sublimación $\left(E_{s}\right)$, según una ecuación de balance del tipo:

$$
\frac{\partial W_{s}}{\partial t}=P_{s}-E_{s}-M_{l t}
$$

La densidad de la nieve también sigue una ecuación de evolución desde una densidad mínima inicial y aumentando exponencialmente con la edad de la nieve hacia una $\rho_{\max }$. Igualmente, el albedo de la nieve, que repercute en los balances de energía entre el suelo y la atmósfera, varía entre 0,5 y 0,85 en ambos modelos, disminuyendo 
exponencial o linealmente en función de si existe o no fusión en la capa. Además, la fracción cubierta de nieve se deriva mediante ecuaciones de diagnóstico. En la tabla 1 se muestran las principales diferencias entre ambas parametrizaciones.

\begin{tabular}{|l|l|l|}
\cline { 2 - 3 } \multicolumn{1}{l|}{} & Harmonie-Arome 40h1.1 (DouvILLE, 1995) & \multicolumn{1}{c|}{ ECMWF (Dutra et al., 2010) } \\
\hline $\begin{array}{l}\text { Agua líquida en } \\
\text { el manto }\end{array}$ & $\begin{array}{l}\text { No se considera agua líquida en la capa } \\
\text { de nieve. La precipitación líquida } \\
\text { "atraviesa» el manto nivoso. }\end{array}$ & $\begin{array}{l}\text { Considera agua líquida en función de la } \\
\text { cantidad total de agua en el manto y su } \\
\text { temperatura. También se tiene en } \\
\text { cuenta la intercepción de lluvia en el } \\
\text { manto. }\end{array}$ \\
\hline $\begin{array}{l}\text { Heterogeneidad } \\
\text { superficial }\end{array}$ & $\begin{array}{l}\text { No distingue entre nieve sobre bosque } \\
\text { y sobre superficie no forestal. El } \\
\text { modelo de suelo realiza un único } \\
\text { balance energético en cada punto } \\
\text { agregando las fracciones cubiertas y } \\
\text { descubiertas de nieve. }\end{array}$ & $\begin{array}{l}\text { El modelo de suelo tiene teselas } \\
\text { diferenciadas para suelo desnudo y } \\
\text { bosque. La nieve expuesta y la nieve } \\
\text { bajo vegetación alta (con menor } \\
\text { albedo) contribuyen por separado al } \\
\text { balance radiativo. }\end{array}$ \\
\hline $\begin{array}{l}\text { Densidad de la } \\
\text { nieve }\end{array}$ & $\begin{array}{l}\text { Toma valores entre } 100 \text { y } 300 \mathrm{~kg} \mathrm{~m}^{-3} \text {. La } \\
\text { «nieve fresca» siempre es igual de } \\
\text { ligera (100 } \mathrm{kg} \mathrm{m}^{-3} \text { ). }\end{array}$ & $\begin{array}{l}\text { Toma valores entre } 100 \text { y } 400 \mathrm{~kg} \mathrm{~m}^{-3} \text {. La } \\
\text { densidad de la nieve «fresca» es } \\
\text { función de la temperatura y la } \\
\text { velocidad del viento. }\end{array}$ \\
\hline
\end{tabular}

Tabla 1. Diferencias entre las parametrizaciones de capa de nieve en los dos modelos.

Además, la asimilación de superficie de ambos modelos también muestra diferencias en lo que respecta a la nieve. En Harmonie-Arome únicamente se asimilan, mediante un algoritmo de interpolación óptima, datos convencionales de espesor de nieve diseminados en mensajes SYNOP. Por su parte, el modelo ECMWF también asimila (igualmente mediante interpolación óptima) los espesores cifrados en los SYNOP, pero añade además el producto IMS de cobertura de nieve (véase sección 3.2), que aunque no cuantifica espesores, permite introducir un mayor número de observaciones de espesor nulo. Es importante mencionar que el número de observatorios españoles que cifran rutinariamente el espesor de nieve es escaso (figura 1), por lo que sería deseable aumentar su número.

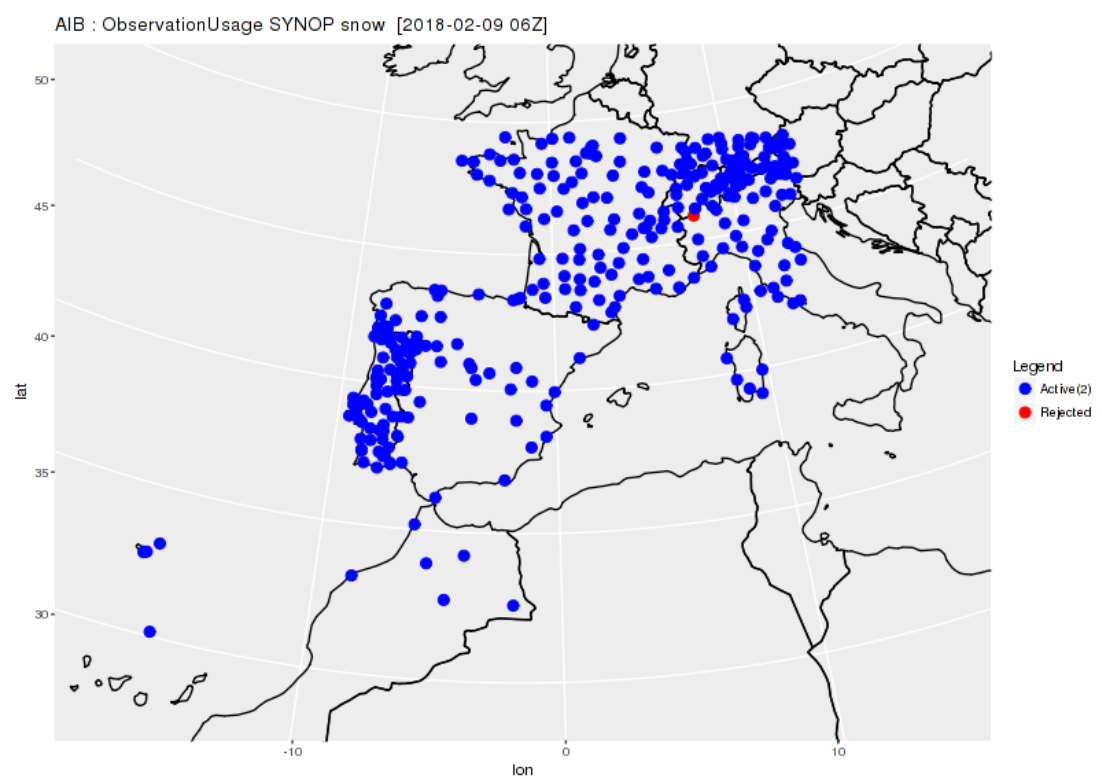

Figura 1. Uso de observaciones de nieve a las $06 \mathrm{Z}$ en la península ibérica y áreas cercanas. 


\section{EJEMPLOS DE VALIDACIÓN DE ESPESORES Y ACUMULACIONES}

\subsection{Validación puntual: estación WMO-SPICE}

La precipitación en forma de nieve es una variable cuya medida plantea dificultades debido a la infraestimación sistemática producida en virtualmente cualquier tipo de pluviómetro según aumenta la velocidad del viento. La Organización Meteorológica Mundial (WMO) ha puesto en marcha diversas iniciativas para mejorar las observaciones automáticas de precipitación sólida, comparar los sesgos sistemáticos de los distintos tipos de instrumentación de medida de precipitación y proponer mejoras para reducirlos. La última de ellas se conoce como proyecto WMO-SPICE (Solid Precipitation Intercomparison Experiment).

AEMET participa en esta iniciativa mediante el campo de pruebas de Formigal-Sarrios, ubicado a $1800 \mathrm{~m}$ de altitud en el Pirineo aragonés (BuIsán et al., 2017). En este emplazamiento (figura 2) se encuentra instalado el sistema de observación de referencia para el experimento de intercomparación, el DFAR (Double Fence Automatic Reference). El DFAR consiste en un pluviómetro automático de pesada (modelo OTT Pluvio $^{2}$ ) ubicado en el centro de un doble vallado concéntrico octogonal de $12 \mathrm{~m}$ y $4 \mathrm{~m}$ de diámetro. El pluviómetro se encuentra rodeado por un escudo de tipo simple y junto con un sensor de tipo de precipitación. La estación de Formigal-Sarrios ocupa un emplazamiento llano a $1800 \mathrm{~m}$ de altitud, no sometido a transporte de nieve, por lo que se considera que ofrece medidas fiables y representativas de acumulaciones y espesores, lo que hace que sean muy aptas para comparar con las salidas de modelos numéricos.

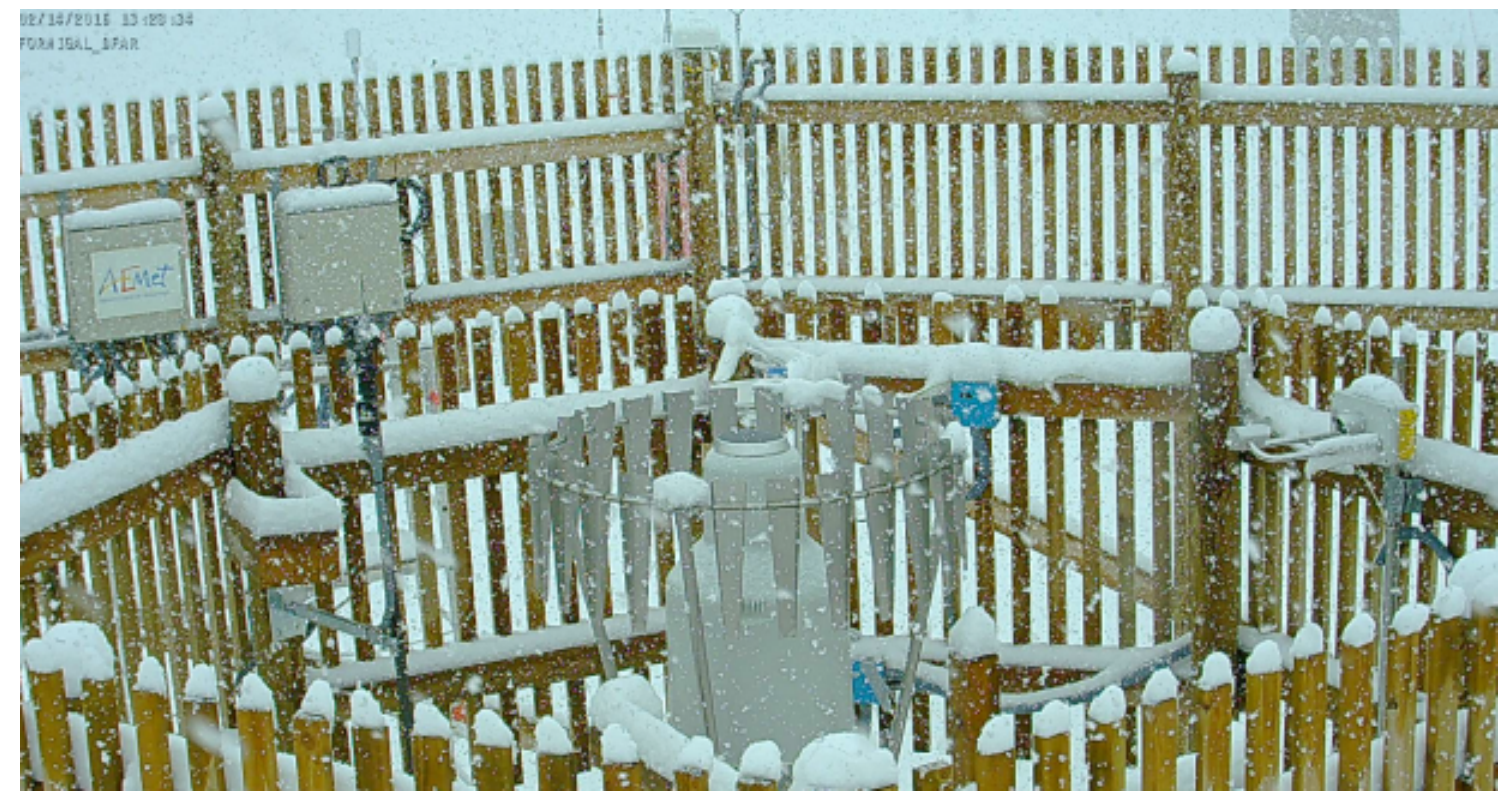

Figura 2. Interior del DFAR de Formigal-Sarrios, con el pluviómetro de pesada y el sensor de tiempo presente (disdrómetro) en el interior del anillo central.

En la figura 3 se puede observar la precipitación total acumulada entre febrero y marzo de 2017 en Formigal-Sarrios según el sistema de referencia DFAR, un pluviómetro estándar como los que figuran en el $80 \%$ de las estaciones automáticas de AEMET, y los pronósticos de Harmonie-Arome y el ECMWF. En estos meses la precipitación fue casi exclusivamente en forma de nieve. Puede observarse cómo las observaciones convencionales subestiman bastante la nieve en zonas montañosas. La salida del ECMWF igualmente subestima, en una proporción similar al pluviómetro estándar. Por su parte, las dos versiones del modelo Harmonie-Arome se acercan bastante más a las acumulaciones del DFAR: no hay muchas diferencias entre los ciclos 38 y 40 de Harmonie-Arome, aunque el primero parece que tendió a producir más precipitación. 


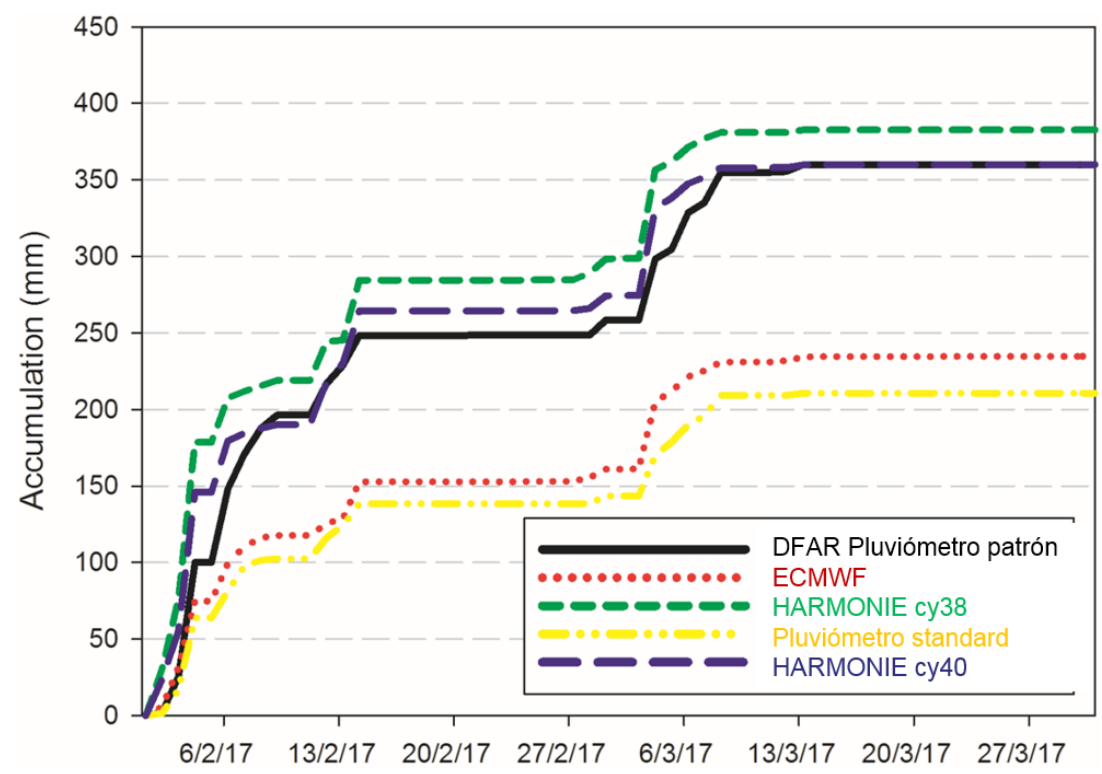

Figura 3. Precipitación acumulada en Formigal-Sarrios según el sistema de referencia DFAR, un pluviómetro estándar, el modelo ECMWF y los ciclos 38 y 40 de Harmonie-Arome.

En cuanto a los espesores de nieve en suelo, la figura 4 muestra cómo Harmonie subestima en general los espesores de nieve en la temporada 2017/18, mientras que el ECMWF sobreestimó las nevadas del inicio de la temporada y cambió la tendencia a partir de entonces, subestimando notablemente las nevadas de primavera y en general fundiendo nieve en exceso.

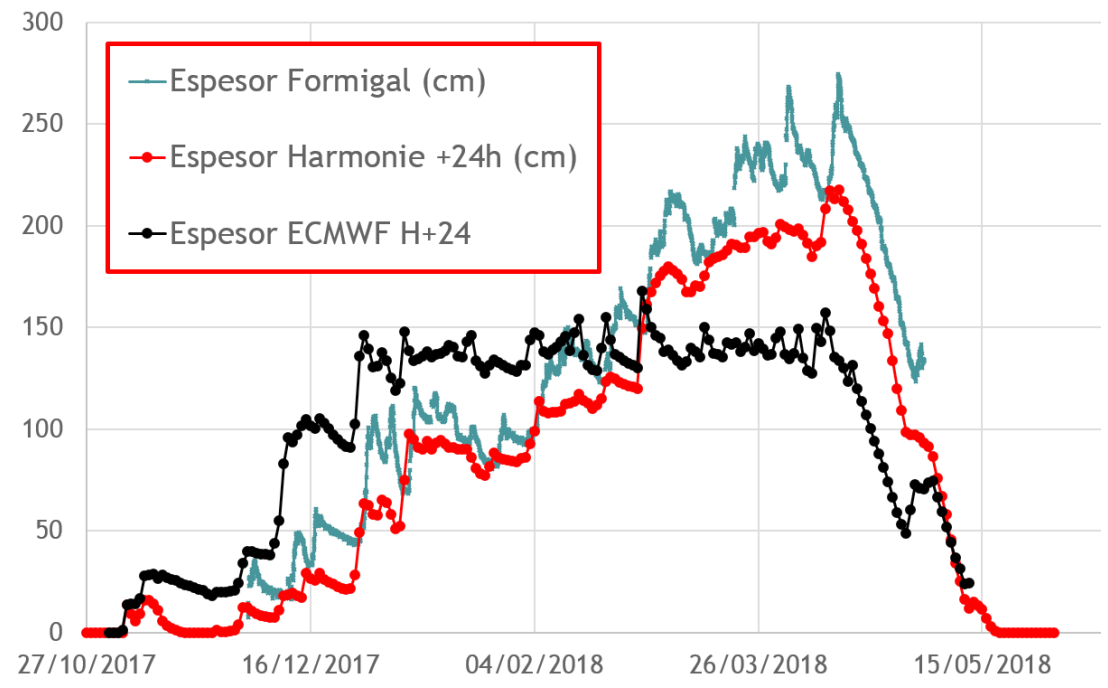

Figura 4. Espesor de nieve en Formigal-Sarrios en la temporada 2017-2018 según el sensor de espesor UDS-Campbell instalado en el emplazamiento, y espesores estimados según los modelos Harmonie-Arome y ECMWF.

\subsection{Comparación con producto IMS de la NOAA}

El NSIDC (National Snow and Ice Data Center) produce un análisis diario de cobertura de nieve a 1, 4 y $24 \mathrm{~km}$ para el hemisferio norte. El IMS o Interactive Multisensor Snow and Ice Mapping System (RAMSAY, 1998) está basado en diversos productos de satélite y observaciones de superficie. En la figura 5 puede observarse cómo la extensión de nieve en Harmonie-Arome tras el episodio de nevadas del 6-8 de enero de 2018 recoge un área mayor y se compara mejor con el producto IMS que en el caso del modelo del Centro 
Europeo. Para el final de la temporada (figura 6) las diferencias entre ambos modelos no son tan grandes y podrían achacarse en parte a la diferencia de resoluciones, pero igualmente las extensiones del Harmonie-Arome se parecen más al producto IMS de alta resolución.
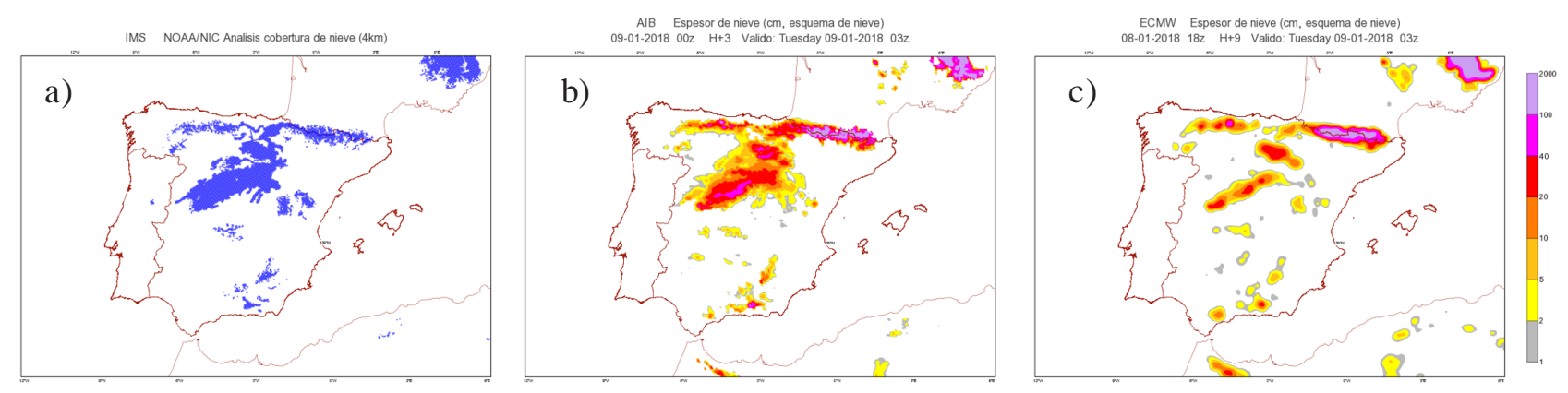

Figura 5. a) Cobertura de nieve IMS y espesores de b) Harmonie-Arome 40 y c) ECMWF para el 9 de enero de 2018.
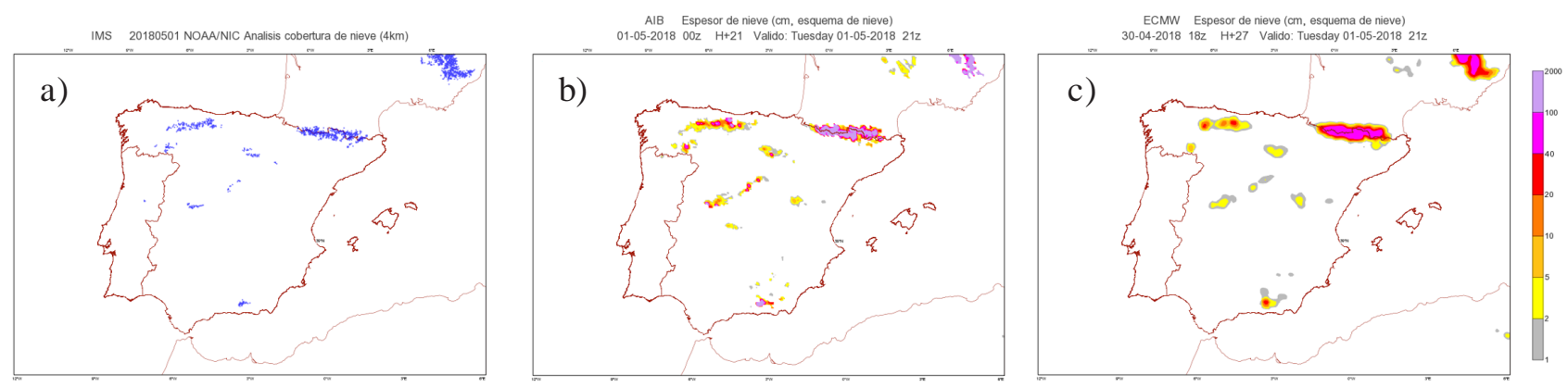

Figura 6. Ídem a la figura 5, para el 5 de mayo de 2018.

\section{LA REPRESENTACIÓN DE LA NIEVE EN FUTURAS VERSIONES DE HARMONIE-AROME}

Actualmente el grupo de trabajo de superficie del consorcio HIRLAM trabaja en aumentar la complejidad de la representación de la superficie incorporando opciones avanzadas disponibles en SURFEX. Para el caso de la nieve, se espera activar el esquema multicapa Explicit Snow (BOONE y ETCHEVERs, 2001), que simula algunos procesos internos del manto nivoso como la transmisión de radiación, procesos de congelacióndescongelación, compactación y estabilización o el almacenamiento de agua líquida y su movimiento entre capas. En este esquema, los intercambios energéticos y de masa con la atmósfera y el suelo quedan mejor representados. Además, algunas otras opciones, no explícitamente de nieve, tendrán también implicaciones en la evolución de la nieve en superficie (pueden consultarse más detalles en el trabajo sobre procesos de superficie en Harmonie-Arome presentado en este mismo Simposio). En cuanto a las mejoras en la asimilación, se espera cambiar el algoritmo de asimilación hacia Ensemble Kalman Filter o similares, e incluir nuevas estimaciones satelitales de cobertura o equivalente acuoso de nieve (productos del H-SAF o similares).

\section{CONCLUSIONES}

Se han descrito las características fundamentales de los esquemas de nieve en superficie de los modelos Harmonie-Arome y ECMWF, subrayando sus semejanzas y diferencias, también en lo relativo a la asimilación de espesores de nieve. Como se ha visto, en España muy pocos observatorios cifran este dato en los SYNOP, por lo que sería recomendable empezar a reportarlo sistemáticamente, al menos a las 06 UTC como hacen otros países. Esto beneficiaría sobre todo a la asimilación del Harmonie-Arome, que no incorpora 
el producto IMS. Además de su utilidad en la asimilación de nieve en el modelo del Centro Europeo, el producto IMS es una herramienta muy buena para seguir la evolución de la cobertura nivosa aunque hay que tener en cuenta que no se actualiza convenientemente con presencia de nubes.

Tanto ECMWF como Harmonie-Arome utilizan esquemas relativamente sencillos para representar la evolución de la nieve pero parece que obtienen unos resultados relativamente buenos. El Centro Europeo parece que subestima la cobertura y da resultados irregulares para el espesor de nieve, mientras que Harmonie-Arome prevé acumulaciones y espesores mucho más parecidos a las observaciones en el emplazamiento de Formigal-Sarrios. En un futuro, se espera utilizar esquemas de análisis que puedan usar los datos de satélite, y parametrizaciones de superficie más sofisticadas que representen mejor las características y evolución del manto nivoso. La discretización del manto en múltiples capas permitirá además obtener información sobre la «historia» del manto nivoso y su estabilidad en cada punto durante la temporada invernal, lo que puede dar pie a un mayor uso de las salidas de Harmonie-Arome fuera del campo estrictamente meteorológico, como por ejemplo su uso para aplicaciones hidrológicas o de predicción de aludes.

\section{AGRADECIMIENTOS}

Este trabajo se ha realizado en el marco de la Red Temática WiPSiS, financiada por el proyecto CGL201681828-REDT/AEI del Gobierno de España.

\section{REFERENCIAS}

Balsamo, G., Beljaars, A., Scipal, K., Viterbo, P., van den Hurk, B., Hirschi, M. y Betts, A. K., 2009. A Revised Hydrology for the ECMWF Model: Verification from Field Site to Terrestrial Water Storage and Impact in the Integrated Forecast System. J. Hydrometeor., 10, 623-643.

Boone, A. y EtcheVers, P., 2001. An intercomparison of three snow schemes of varying complexity coupled to the same land surface model: Local-scale evaluation at an alpine site. J. Hydrometeorol., 2 (4), 374-394.

Buisán, S. T., Earle, M.E., Collado, J. L., Kochendorfer, J., Alastrué, J., Wolff, M., Smith, C. D. y LóPEZ-Moreno, J. I., 2017. Assessment of snowfall accumulation underestimation by tipping bucket gauges in the Spanish operational network. Atmos. Meas. Tech., 10, 1079-1091.

Douville, H., Royer, J. F. y Mahfouf, J. F., 1995. A New Snow Parameterization for the Meteo-France Climate Model. Validation in Stand-Alone Experiments. Climate Dyn., 12, 21-35.

Dutra, E., Balsamo, G., Viterbo, P., Miranda, P. M. A., Beljaars, A., Schar, C. y Elder, K., 2010. An improved snow scheme for the ECMWF land surface model: Description and offline validation. J. Hydrometeorol., 11, 899-916.

Masson, V., Le Moigne, P., Martin, E., Faroux, S., Alias, A., Alkama, R., Belamari, S., Barbu, A., Boone, A., Bouyssel, F., Brousseau, P., Brun, E., Calvet, J.-C., Carrer, D., Decharme, B., Delire, C., Donier, S., Essaouini, K., Gibelin, A.-L., Giordani, H., Habets, F., Jidane, M., Kerdraon, G., Kourzeneva, E., Lafaysse, M., Lafont, S., Lebeaupin Brossier, C., Lemonsu, A., Mahfouf, J.-F., Marguinaud, P., Mokhtari, M., Morin, S., Pigeon, G., Salgado, R., Seity, Y., Taillefer, F., Tanguy, G., Tulet, P., Vincendon, B., Vionnet, V. y Voldoire, A., 2013. The SURFEXv7.2 land and ocean surface platform for coupled or offline simulation of earth surface variables and fluxes. Geosci. Model Dev., 6, 929-960.

Ramsay, B. H., 1998. The interactive multisensor snow and ice mapping system. Hydrol. Process., 12, 1537-1546. 